\title{
The Cayley isomorphism property for groups of order $8 p$
}

\author{
Gábor Somlai * \\ Eötvös Loránd University, Department of Algebra and Number Theory, \\ 1117 Pázmány Péter sétány 1/C , Budapest, Hungary
}

Received 31 December 2013, accepted 10 September 2014, published online 26 June 2015

\begin{abstract}
For every prime $p>3$ we prove that $Q \times \mathbb{Z}_{p}$ is a DCI-group, where $Q$ denotes the quaternion group of order 8 . Using the same method we reprove the fact that $\mathbb{Z}_{2}^{3} \times \mathbb{Z}_{p}$ is a CI-group for every prime $p>3$, which was obtained in [3]. This result completes the description of CI-groups of order $8 p$.
\end{abstract}

Keywords: Cayley graphs, CI-groups.

Math. Subj. Class.: 05C25

\section{Introduction}

Let $G$ be a finite group and $S$ a subset of $G$. The Cayley graph $\operatorname{Cay}(G, S)$ is defined by having the vertex set $G$ and $g$ is adjacent to $h$ if and only if $g^{-1} h \in S$. The set $S$ is called the connection set of the Cayley graph $\operatorname{Cay}(G, S)$. A Cayley graph $\operatorname{Cay}(G, S)$ is undirected if and only if $S=S^{-1}$, where $S^{-1}=\left\{s^{-1} \in G \mid s \in S\right\}$. Every left multiplication via elements of $G$ is an automorphism of $\operatorname{Cay}(G, S)$, so the automorphism group of every Cayley graph on $G$ contains a regular subgroup isomorphic to $G$. Moreover, this property characterises the Cayley graphs of $G$.

Similarly to Cayley graphs one can also define ternary Cayley relational structures. $\left(V, E_{1}, E_{2}, \ldots, E_{l}\right)$ is a colour ternary relational structure if $E_{i} \subset V^{3}$ for $i=1, \ldots, l$. We say that a colour ternary relational structure $\left(V, E_{1}, \ldots, E_{l}\right)$ is a Cayley ternary relational structure of the group $G$ if the automorphism group of $\left(V, E_{1}, \ldots, E_{l}\right)$ contains a regular subgroup isomorphic to $G$.

It is clear that $\operatorname{Cay}(G, S) \cong \operatorname{Cay}(G, \mu(S))$ for every $\mu \in \operatorname{Aut}(G)$. A Cayley graph $\operatorname{Cay}(G, S)$ is said to be a CI-graph if, for each $T \subset G$, the Cayley graphs $\operatorname{Cay}(G, S)$

* Research supported by the Hungarian Scientific Fund (OTKA), grant no. K84233

E-mail address: gsomlai@cs.elte.hu (Gábor Somlai) 
and $\operatorname{Cay}(G, T)$ are isomorphic if and only if there is an automorphism $\mu$ of $G$ such that $\mu(S)=T$. Furthermore, a group $G$ is called a DCI-group if every Cayley graph of $G$ is a CI-graph and it is called a CI-group if every undirected Cayley graph of $G$ is a CI-graph.

Similarly, a group $G$ is called a CI-group with respect to colour ternary relational structures, if for any pair of isomorphic colour ternary relational structures of $G$ there exists an isomorphism induced by an automorphism of $G$.

Let $G$ be a CI-group of order $8 p$, where $p$ is an odd prime. It is easy to verify that $\mathbb{Z}_{2} \times \mathbb{Z}_{4}$ and the dihedral group of order 8 are not CI-groups. It can easily be seen that every subgroup of a CI-group is also a CI-group. Therefore the Sylow 2-subgroup of $G$ can only be $\mathbb{Z}_{8}, \mathbb{Z}_{2}^{3}$ or the quaternion group $Q$ of order 8 .

It was proved by Li, Lu and Pálfy [5, Theorem 1.2 (b)] that a finite CI-group of order $8 p$ containing an element of order 8 can only be

$$
H=\left\langle a, z \mid a^{p}=1, z^{8}=1, z^{-1} a z=a^{-1}\right\rangle .
$$

It was also shown in [5, Theorem 1.3] that $H$ is a CI-group, though not a DCI-group. In view of these results, for the rest of the discussion, we assume that the Sylow 2-subgroup of $G$ is isomorphic to $Q$ or $\mathbb{Z}_{2}^{3}$.

It was proved by Dobson [2] that $\mathbb{Z}_{2}^{3} \times \mathbb{Z}_{p}$ is a CI-group with respect to ternary relational structures if $p \geq 11$. Moreover, Dobson and Spiga [3] proved that $\mathbb{Z}_{2}^{3} \times \mathbb{Z}_{p}$ is a DCIgroup with respect to colour ternary relational structures if and only if $p \neq 3$ and 7 . As a consequence of this result it was proved in [3] that $\mathbb{Z}_{2}^{3} \times \mathbb{Z}_{p}$ is a DCI-group for all primes $p$.

If $p>8$ or $p=5$, then by Sylow's Theorem the Sylow $p$-subgroup of $G$ is a normal subgroup, therefore $G$ is isomorphic to one of the following groups: $\mathbb{Z}_{2}^{3} \times \mathbb{Z}_{p}, Q \times \mathbb{Z}_{p}$, $\mathbb{Z}_{2}^{3} \ltimes \mathbb{Z}_{p}$ or $Q \ltimes \mathbb{Z}_{p}$. It can also be seen from [5, Theorem 1.2] that neither $Q \ltimes \mathbb{Z}_{p}$ nor $\mathbb{Z}_{2}^{3} \ltimes \mathbb{Z}_{p}$ is a CI-group.

If $p=7$, then either the Sylow 7-subgroup is normal, in which case $G$ is as before, or $G$ has 8 Sylow 7 -subgroups and the Sylow 2 -subgroup of $G$ is normal. Then the Sylow 7 -subgroup of $G$ acts transitively by conjugation on the the non-identity elements of the Sylow 2-subgroup. Hence $G \cong \mathbb{Z}_{2}^{3} \rtimes \mathbb{Z}_{7}$, which is not a CI-group by [5, Theorem 1.2.(b)].

If $p=3$, then the order of $G$ is 24 . A complete list of CI-groups of order at most 31 was given in the Ph.D. thesis of Royle, see [7]. The CI-groups of order 24 are the following: $Q \times \mathbb{Z}_{3}, \mathbb{Z}_{8} \ltimes \mathbb{Z}_{3}$ and $\mathbb{Z}_{2}^{3} \times \mathbb{Z}_{3}$.

Spiga [6] proved that $Q \times \mathbb{Z}_{3}$ is not a CI-group with respect to colour ternary relational structures.

Using different methods depending on whether $p>8$, or $p=5,7$ we show that the other groups are DCI-groups. By extending our result with the fact that $\mathbb{Z}_{2}^{3} \times \mathbb{Z}_{3}$ is a CI-group we get that $Q \times \mathbb{Z}_{p}$ is a CI-group for every odd prime $p$.

Theorem 1.1. For every prime $p \geq 3$ the group $Q \times \mathbb{Z}_{p}$ is a DCI-group.

We also prove the following result which was first obtained in [3].

Theorem 1.2 (Dobson, Spiga [3]). For every prime $p \geq 3$ the group $\mathbb{Z}_{2}^{3} \times \mathbb{Z}_{p}$ is a DCIgroup.

Our paper is organized as follows. In Section 2 we introduce the notation that will be used throughout this paper. In Section 3 we collect important ideas which are useful in the proof of Theorem 1.1 and 1.2. Section 4 contains the proof of Theorem 1.1 and 1.2 for primes $p>8$ and Section 5 contains the proof of Theorem 1.1 and 1.2 for $p=5$ and 7 . 


\section{Technical details}

In this section we introduce some notation. Let $G$ be a group. We use $H \leq G$ to denote that $H$ is a subgroup of $G$ and by $N_{G}(H)$ and $C_{G}(H)$ we denote the normalizer and the centralizer of $H$ in $G$, respectively.

Let us assume that the group $H$ acts on the set $\Omega$ and let $G$ be an arbitrary group. Then by $G \imath_{\Omega} H$ we denote the wreath product of $G$ and $H$. Every element $g \in G \imath_{\Omega} H$ can be uniquely written as $h k$, where $k \in K=\prod_{\omega \in \Omega} G_{\omega}$ and $h \in H$. The group $K=\prod_{\omega \in \Omega} G_{\omega}$ is called the base group of $G 2_{\Omega} H$ and the elements of $K$ can be treated as functions from $\Omega$ to $G$. If $g \in G \imath_{\Omega} H$ and $g=h k$ we denote $k$ by $(g)_{b}$. In order to simplify the notation $\Omega$ will be omitted if it is clear from the definition of $H$ and we will write $G$ ? $H$.

The symmetric group on the set $\Omega$ will be denoted by $\operatorname{Sym}(\Omega)$. Let $G$ be a permutation group on the set $\Omega$. For a $G$-invariant partition $\mathcal{B}$ of the set $\Omega$ we use $G^{\mathcal{B}}$ to denote the permutation group on $\mathcal{B}$ induced by the action of $G$ and similarly, for every $g \in G$ we denote by $g^{\mathcal{B}}$ the action of $g$ on the partition $\mathcal{B}$.

For a group $G$, let $\hat{G}$ denote the subgroup of the symmetric group $\operatorname{Sym}(G)$ formed by the elements of $G$ acting by right multiplication on $G$. For every Cayley graph $\Gamma=$ $\operatorname{Cay}(G, S)$ the subgroup $\hat{G}$ of $\operatorname{Sym}(G)$ is contained in $\operatorname{Aut}(\Gamma)$.

Definition 2.1. Let $G \leq \operatorname{Sym}(\Omega)$ be a permutation group. Let

$$
G^{(2)}=\left\{\pi \in \operatorname{Sym}(\Omega) \mid \forall a, b \in \Omega \exists g_{a, b} \in G \text { with } \begin{array}{c}
\pi(a)=g_{a, b}(a) \text { and } \\
\pi(b)=g_{a, b}(b)
\end{array}\right\} .
$$

We say that $G^{(2)}$ is the 2-closure of the permutation group $G$.

The following lemma is well-known and follows directly from the definition of $G^{(2)}$.

Lemma 2.2. Let $\Gamma$ be a graph. If $G \leq A u t(\Gamma)$, then $G^{(2)} \leq \operatorname{Aut}(\Gamma)$.

\section{Basic ideas}

In this section we collect some results and some important ideas that we will use in the proof of Theorem 1.1 and Theorem 1.2.

We begin with a fundamental lemma that we will use all along this paper.

Lemma 3.1 (Babai [1]). The Cayley graph Cay $(G, S)$ is a CI-graph if and only iffor every regular subgroup $\stackrel{G}{G}$ of $\operatorname{Aut}(\operatorname{Cay}(G, S))$ isomorphic to $G$ there is a $\mu \in \operatorname{Aut}(\operatorname{Cay}(G, S))$ such that $\stackrel{\circ}{G}^{\mu}=\hat{G}$.

We introduce the following definition.

Definition 3.2. (a) We say that a Cayley graph $\operatorname{Cay}(G, S)$ is a $\mathrm{CI}^{(2)}$-graph if and only if for every regular subgroup $\stackrel{\circ}{G}$ of $\operatorname{Aut}(\operatorname{Cay}(G, S))$ isomorphic to $G$ there is a $\sigma \in$ $\langle\stackrel{\leftrightarrow}{G}, \hat{G}\rangle^{(2)}$ such that $\stackrel{\circ}{G}^{\sigma}=\hat{G}$.

(b) A group $G$ is called a $\mathrm{DCI}^{(2)}$-group if for every $S \subset G$ the Cayley graph $\operatorname{Cay}(G, S)$ is a $\mathrm{CI}^{(2)}$-graph.

Let $R$ be either $Q$ or $\mathbb{Z}_{2}^{3}$. Let us assume that $A=\operatorname{Aut}(\operatorname{Cay}(G, S)) \leq \operatorname{Sym}(8 p)$ contains two copies of regular subgroups, $\stackrel{\circ}{R} \times \stackrel{\circ}{\mathbb{Z}}_{p}$ and $\hat{R} \times \hat{\mathbb{Z}}_{p}$. By Sylow's theorem we may assume that $\stackrel{\mathbb{Z}}{p}_{p}$ and $\hat{\mathbb{Z}}_{p}$ are in the same Sylow $p$-subgroup $P$ of $\operatorname{Sym}(8 p)$. If $p>8$, 
then $P$ is isomorphic to $\mathbb{Z}_{p}^{8}$. Moreover, $P$ is generated by 8 disjoint $p$-cycles. It follows that both $\stackrel{\circ}{R}$ and $\hat{R}$ normalize $P$ so we may assume that $\stackrel{\circ}{R}$ and $\hat{R}$ lie in the same Sylow 2subgroup of $N_{A}(P)$. Let $P_{2}$ denote a Sylow 2-subgroup of $\operatorname{Sym}(8)$. It is also well known that $P_{2}$ is isomorphic to the automorphism group of the following graph $\Delta$ :

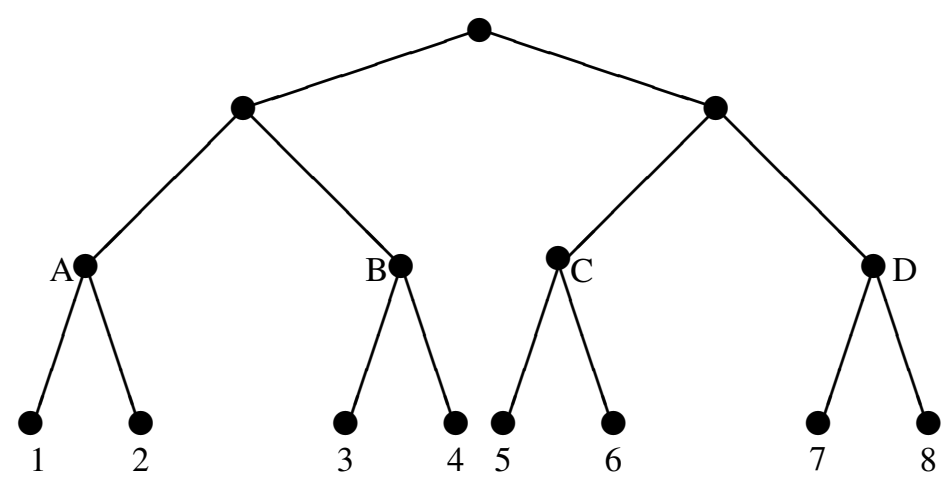

Every automorphism of $\Delta$ permutes the leaves of the graph and the permutation of the leaves determines the automorphism, therefore $A u t(\Delta)$ can naturally be embedded into $\operatorname{Sym}(8)$.

Lemma 3.3. (a) There are exactly two regular subgroups of $P_{2}$ which are isomorphic to $Q$.

(b) There are exactly two regular subgroups of $P_{2}$ which are isomorphic to $\mathbb{Z}_{2}^{3}$.

Proof. (a) Let $Q$ be a regular subgroup of $A u t(\Delta)$ isomorphic to the quaternion group with generators $i$ and $j$. Since $Q$ is regular, for every $1 \leq m \leq 4$ there is a $q_{m} \in Q$ (not necessarily distinct) such that $q_{m}(2 m-1)=2 m$. These are automorphisms of $\Delta$ so $q_{m}(2 m)=2 m-1$ and hence since $Q$ is regular the order of $q_{m}$ is 2 . There is only one involution in $Q$ so $q_{m}=i^{2}$ for every $1 \leq m \leq 4$ and this fact determines completely the action of $i^{2}$ on $\Delta$. Note that the automorphisms $q_{m}$ are all equal.

We can assume that $i(1)=3$. Such an isomorphism of $\Delta$ fixes setwise $\{1,2,3,4\}$ so we have that $i(3)=2, i(2)=4$ and $i(4)=1$ since $i$ is of order 4 . Using again the fact that $Q$ is regular on $\Delta$ and $i^{2}(5)=6$, we get that there are two choices for the action of $i: i=(1324)(5768)$ or $i=(1324)(5867)$.

We can also assume that $j(1)=5$. This implies that $j(5)=j^{2}(1)=i^{2}(1)=2$, and $j(2)=6$ since $j \in \operatorname{Aut}(\Delta)$ and $j(6)=1$. The action of $i$ determines the action of $j$ on $\Delta$ since $i j i=j$. Applying this to the leaf 3 we get that $j(3)=8$ if $i=(1324)(5768)$ and $j(3)=7$ if $i=(1324)(5867)$ so there is no more choice for the action of $j$. Finally, $i$ and $j$ generate $Q$ and this gives the result.

(b) One can prove this using an argument similar to the previous case.

The previous proof also gives the following. 
Lemma 3.4. (a) The following two pairs of permutations generate the two regular subgroups of $A u t(\Delta) \leq \operatorname{Sym}(8)$ isomorphic to $Q$ :

$$
\begin{aligned}
& i_{1}=(1324)(5768), j_{1}=(1526)(3748) \text { and } \\
& i_{2}=(1324)(5867), j_{2}=(1526)(3847) .
\end{aligned}
$$

(b) The elements of these regular subgroups of $A u t(\Delta)$ are the following:

\begin{tabular}{cc|cc}
$Q_{l}:$ & & $Q_{r}:$ & \\
\hline id & $(12)(34)(56)(78)$ & $i d$ & $(12)(34)(56)(78)$ \\
$(1324)(5768)$ & $(1423)(5867)$ & $(1324)(5867)$ & $(1423)(5768)$ \\
$(1526)(3847)$ & $(1625)(3748)$ & $(1526)(3748)$ & $(1625)(3847)$ \\
$(1728)(3546)$ & $(1827)(3645)$ & $(1728)(3645)$ & $(1827)(3546)$
\end{tabular}

Using the identification given in the following table, $Q_{l}$ and $Q_{r}$ act on $Q$ by left-and right-multiplication with the elements of $Q$, respectively:

$$
\begin{array}{c||c|c|c|c|c|c|c|c}
\{1, \ldots, 8\} & 1 & 2 & 3 & 4 & 5 & 6 & 7 & 8 \\
Q & 1 & -1 & i & -i & j & -j & k & -k
\end{array} .
$$

(c) $A_{1}=\left\langle x_{1}, x_{2}, x_{3}\right\rangle$ and $A_{2}=\left\langle y_{1}, y_{2}, y_{3}\right\rangle$ are subgroups of $\operatorname{Aut}(\Delta) \leq \operatorname{Sym}(8)$ isomorphic to $\mathbb{Z}_{2}^{3}$, where

$$
\begin{aligned}
& x_{1}=(12)(34)(56)(78), x_{2}=(13)(24)(57)(68), x_{3}=(15)(26)(37)(48) \text { and } \\
& y_{1}=(12)(34)(56)(78), y_{2}=(13)(24)(58)(67), y_{3}=(15)(26)(38)(47) .
\end{aligned}
$$

Lemma 3.5. Let us assume that $G_{1} \leq P_{2}$ is generated by two different regular subgroups $Q_{a}$ and $Q_{b}$ of $A u t(\Delta)$ which are isomorphic to $Q$ and $G_{2} \leq P_{2}$ is generated by two different regular subgroups $A_{1}$ and $A_{2}$ of $A u t(\Delta)$ which are isomorphic to $\mathbb{Z}_{2}^{3}$. Then $G_{1}=G_{2}$.

Proof. It is clear that $\left|P_{2}\right|=|A u t(\Delta)|=2^{7}$. One can see using Lemma 3.4 (a) and (c) that $G_{1}$ and $G_{2}$ are generated by even permutations. Both $G_{1}$ and $G_{2}$ induce an action on the set $V=\{A, B, C, D\}$ which is a set of vertices of $\Delta$ and it is easy to verify that every permutation of $V$ induced by $G_{1}$ and $G_{2}$ is even. This shows that $G_{1}$ and $G_{2}$ are contained in a subgroup of $P_{2}$ of cardinality $2^{5}$.

Lemma 3.4 (b) shows that $\left|Q_{a} \cap Q_{b}\right|=2$ and one can also check using Lemma 3.4 (c) that $\left|A_{1} \cap A_{2}\right|=2$. This gives $\left|G_{1}\right| \geq 2^{5}$ and $\left|G_{2}\right| \geq 2^{5}$, finishing the proof of Lemma 3.5.

Proposition 3.6. (a) The quaternion group $Q$ is a $D C I^{(2)}$-group.

(b) The elementary abelian group $\mathbb{Z}_{2}^{3}$ is a $D C I^{(2)}$-group.

Proof. (a) Let $Q_{a}$ and $Q_{b}$ be two regular subgroups of $\operatorname{Sym}(8)$ isomorphic to the quaternion group $Q$. By Sylow's theorem we may assume that $Q_{a}$ and $Q_{b}$ lie in the same Sylow 2-subgroup of $H=\left\langle Q_{a}, Q_{b}\right\rangle$. Since every Sylow 2-subgroup of $H$ is contained in a Sylow 2-subgroup of $\operatorname{Sym}(8)$, we may assume that $Q_{a}$ and $Q_{b}$ are subgroups of $\operatorname{Aut}(\Delta)$. 
Our aim is to find an element $\pi \in\left\langle Q_{a}, Q_{b}\right\rangle^{(2)}$ such that $Q_{a}^{\pi}=Q_{b}$. Let us assume that $Q_{a} \neq Q_{b}$. Using Lemma 3.4 (a) we may also assume that $Q_{a}$ and $Q_{b}$ are generated by the permutations $(1324)(5768),(1526)(3748)$ and (1324)(5867), (1526)(3847), respectively. Lemma 3.4 (b) shows that $H$ contains the following three permutations:

$$
\begin{aligned}
& (12)(34)=(1324)(5768)(1324)(5867) \\
& (12)(56)=(1526)(3748)(1526)(3847) \\
& (12)(78)=(1728)(3546)(1728)(3645) .
\end{aligned}
$$

Now one can easily see that the permutation (12) is in $H^{(2)}$. Finally, it is also easy to check using Lemma 3.4 (b) that $Q_{a}^{(12)}=Q_{b}$.

(b) One can prove this statement using Lemma 3.4 and Lemma 3.5.

Definition 3.7. Let $\Gamma$ be an arbitrary graph and $A, B \subset V(\Gamma)$ such that $A \cap B=\emptyset$. We write $A \sim B$ if one of the following four possibilities holds:

(a) For every $a \in A$ and $b \in B$ there is an edge from $a$ to $b$ but there is no edge from $b$ to $a$.

(b) For every $a \in A$ and $b \in B$ there is an edge from $b$ to $a$ but there is no edge from $a$ to $b$.

(c) For every $a \in A$ and $b \in B$ the vertices $a$ and $b$ are connected with an undirected edge.

(d) There is no edge between $A$ and $B$.

We also write $A \nsim B$ if none of the previous four possibilities holds.

The following lemma follows easily:

Lemma 3.8. Let $A, B$ be two disjoint subsets of cardinality $p$ of a graph. We write $A \cup B=$ $\mathbb{Z}_{p} \cup \mathbb{Z}_{p}$. Let us assume that a generator $\hat{g}$ of $\hat{\mathbb{Z}}_{p}$ acts by $\hat{g}\left(a_{1}, a_{2}\right)=\left(a_{1}+1, a_{2}+1\right)$ on $A \cup B$ and for a generator $\stackrel{\circ}{a}$ of the cyclic group $\stackrel{\circ}{\mathbb{Z}}_{p}$ the action of $\stackrel{\circ}{a}$ is defined by $\stackrel{\circ}{a}\left(a_{1}, a_{2}\right)=$ $\left(a_{1}+b, a_{2}+c\right)$ for some $b, c \in \mathbb{Z}_{p}$.

(a) If $b=c$, then the action of $\hat{\mathbb{Z}}_{p}$ and $\stackrel{Z}{Z}_{p}$ on $A \cup B$ are the same.

(b) If $A \nsim B$, then $b=c$.

(c) If $A \sim B$, then every $\pi \in S y m(A \cup B)$ which fixes $A$ and $B$ setwise is an automorphism of the graph defined on $A \cup B$ as long as $\left.\pi\right|_{A} \in A u t(A)$ and $\left.\pi\right|_{B} \in A u t(B)$.

\section{Main result for $p>8$}

In this section, we will prove that $R \times \mathbb{Z}_{p}$ is a DCI-group if $p>8$, where $R$ is either $Q$ or $\mathbb{Z}_{2}^{3}$.

Proposition 4.1. For every prime $p>8$, the group $R \times \mathbb{Z}_{p}$ is a DCI-group.

Our technique is based on Lemma 3.1 so we have to fix a Cayley graph $\Gamma=\operatorname{Cay}(R \times$ $\left.\mathbb{Z}_{p}, S\right)$. Let $A=A u t(\Gamma)$ and $\stackrel{\circ}{G}=\stackrel{\circ}{R} \times \stackrel{\circ}{\mathbb{Z}}_{p}$ be a regular subgroup of $A$ isomorphic to $R \times \mathbb{Z}_{p}$. In order to prove Proposition 4.1 we have to find an $\alpha \in A$ such that $\stackrel{\circ}{G}^{\alpha}=\hat{G}=\hat{R} \times \hat{\mathbb{Z}}_{p}$. We will achieve this in three steps. 


\subsection{Step 1}

Since $p>8$, the Sylow $p$-subgroup of $\operatorname{Sym}(8 \mathrm{p})$ is generated by 8 disjoint $p$-cycles. We may assume $\hat{\mathbb{Z}}_{p}$ and $\stackrel{\mathbb{Z}}{p}_{p}$ lie in the same Sylow p-subgroup $P$ of $\operatorname{Sym}(8 p)$. Then both $\stackrel{\circ}{R}$ and $\hat{R}$ are subgroups of $N_{S y m(8 p)}(P) \cap A$ so we may assume that $\stackrel{\circ}{R}$ and $\hat{R}$ lie in the same Sylow 2-subgroup of $N_{\text {Sym }(8 p)}(P) \cap A$ which is contained in a Sylow 2-subgroup of $A$.

Since $p>8$, the Sylow $p$-subgroup $P$ gives a partition $\mathcal{B}=\left\{B_{1}, B_{2}, \ldots, B_{8}\right\}$ of the vertices of $\Gamma$, where $\left|B_{i}\right|=p$ for every $i=1, \ldots, 8$ and $\mathcal{B}$ is $P$-invariant. It is easy to see that $\mathcal{B}$ is invariant under the action of $\hat{R}$ and $\stackrel{\circ}{R}$ and hence $\langle\hat{G}, \stackrel{\circ}{G}\rangle \leq \operatorname{Sym}(p)$ < Sym $(8)$. Moreover, both $\stackrel{\circ}{G}$ and $\hat{G}$ are regular so $\stackrel{\circ}{R}$ and $\hat{R}$ induce regular action on $\mathcal{B}$ which we denote by $R_{1}$ and $R_{2}$, respectively. The assumption that $\stackrel{\circ}{R}$ and $\hat{R}$ lie in the same Sylow 2-subgroup of $A$ implies that $R_{1}$ and $R_{2}$ are in the same Sylow 2-subgroup of $\operatorname{Sym}(8)$.

\subsection{Step 2}

Let us assume that $R_{1} \neq R_{2}$. We intend to find an element $\alpha \in A$ such that $\left(\stackrel{\circ}{R}^{\alpha}\right)^{\mathcal{B}}=R_{2}$.

We define a graph $\Gamma_{0}$ on $\mathcal{B}$ such that $B_{m}$ is adjacent to $B_{n}$ if and only if $B_{m} \nsim B_{n}$. This is an undirected graph with vertex set $\mathcal{B}$ and both $R_{1}$ and $R_{2}$ are regular subgroups of $\operatorname{Aut}\left(\Gamma_{0}\right)$. It follows that $\Gamma_{0}$ is a Cayley graph of $R$.

Observation 4.1. Since $R_{1} \leq \operatorname{Aut}\left(\Gamma_{0}\right)$ acts transitively on $\mathcal{B}$ we have that the order of eah connected component of $\Gamma_{0}$ divides 8 .

We can also define a coloured graph $\Gamma_{1}$ on $\mathcal{B}$ by colouring the edges of the complete directed graph on 8 vertices. The vertex $B_{m}$ is adjacent to the vertex $B_{n}$ with the same coloured edge as $B_{m^{\prime}}$ is adjacent to $B_{n^{\prime}}$ in $\Gamma_{1}$ if and only if there exists a graph isomorphism $\phi$ from the induced subgraph of $\Gamma$ on $B_{m} \cup B_{n}$ to the induced subgraph of $\Gamma$ on $B_{m^{\prime}} \cup B_{n^{\prime}}$ such that $\phi\left(B_{m}\right)=B_{m^{\prime}}$ and $\phi\left(B_{n}\right)=B_{n^{\prime}}$. The graph $\Gamma_{1}$ is a coloured Cayley graph of $R$. Moreover, both $R_{1}$ and $R_{2}$ act regularly on $\Gamma_{1}$. Using the fact that $R$ has property $D C I^{(2)}$, it is clear that there exists an $\alpha^{\prime} \in\left\langle R_{1}, R_{2}\right\rangle^{(2)} \leq \operatorname{Aut}\left(\Gamma_{1}\right)$ such that $R_{2}^{\alpha^{\prime}}=R_{1}$. We would like to lift $\alpha^{\prime}$ to an automorphism $\alpha$ of $\Gamma$ such that $\alpha^{\mathcal{B}}=\alpha^{\prime}$.

(a) Let us assume first that $\Gamma_{0}$ is a connected graph.

Lemma 4.2. (a) $\stackrel{\circ}{R} \times \stackrel{\circ}{\mathbb{Z}}_{p} \leq \hat{\mathbb{Z}}_{p}\{\operatorname{Sym}(8)$.

(b) If $\stackrel{\circ}{R} \times \stackrel{\circ}{\mathbb{Z}}_{p} \leq \hat{\mathbb{Z}}_{p}$ L Sym(8), then for every $\stackrel{\circ}{\mathrm{S}} \in \stackrel{\circ}{R}$ we have $(\stackrel{\circ}{r})_{b}=i d$.

Proof. (a) We first prove that $\hat{\mathbb{Z}}_{p}=\stackrel{\circ}{\mathbb{Z}}_{p}$. Let $x$ and $y$ generate $\hat{\mathbb{Z}}_{p}$ and $\stackrel{\mathbb{Z}}{p}_{p}$, respectively. Since $x$ and $y$ lie in the same Sylow $p$-subgroup and $\left|B_{1}\right|=p$, we can assume that $\left.x\right|_{B_{1}}=\left.y\right|_{B_{1}}$. Using Lemma 3.8(b) we get that $\left.x\right|_{B_{m}}=\left.y\right|_{B_{n}}$ if there exists a path in $\Gamma_{0}$ from $B_{m}$ to $B_{n}$. This shows that $x=y$ since $\Gamma_{0}$ is connected. Moreover, $\stackrel{\circ}{R} \times \stackrel{\circ}{\mathbb{Z}}_{p} \leq \hat{\mathbb{Z}}_{p} \imath \operatorname{Sym}(8)$ since the elements of $\stackrel{\circ}{\mathbb{Z}}_{p}$ and the elements of $\stackrel{\circ}{R}$ commute.

(b) Let $A^{\prime}=A \cap\left(\hat{\mathbb{Z}}_{p} 2 \operatorname{Sym}(8)\right)$. We have already assumed that $\stackrel{\circ}{R}$ and $\hat{R}$ lie in the same Sylow 2-subgroup of $A^{\prime}$. Let $\stackrel{\circ}{r}$ be an arbitrary element of $\stackrel{\circ}{R}$. For every $(a, u) \in R \times \mathbb{Z}_{p}$ we have $\stackrel{r}{r}(a, u)=(b, u+t)$ for some $b \in R$ and $t \in \mathbb{Z}_{p}$, where $t$ only depends on $\stackrel{\circ}{r}$ and $a$ since $\stackrel{\circ}{r} \leq \hat{\mathbb{Z}}_{p} \prec \operatorname{Sym}(8)$. The permutation group $\hat{G}$ is transitive, hence there exist $\hat{r}_{1}, \hat{r}_{2} \in \hat{R}$ such that $\hat{r}_{1}(1, u)=(a, u)$ 
and $\hat{r}_{2}(b, u+t)=(1, u+t)$. The order of $\hat{r}_{2} \stackrel{\circ}{r} \hat{r}_{1}$ is a power of 2 since $\hat{r}_{2}, \stackrel{\circ}{r}, \hat{r}_{1}$ lie in a Sylow 2-subgroup. Therefore $t=0$ and hence $(\stackrel{\circ}{r})_{b}=i d$.

Lemma 4.2 says that if $\Gamma_{0}$ is connected, then $\langle\stackrel{\circ}{R}, \hat{R}\rangle \leq \hat{\mathbb{Z}}_{p}\left\langle\operatorname{Sym}(8)\right.$ and $(r)_{b}=i d$ for every $r \in\langle\stackrel{\circ}{R}, \hat{R}\rangle$. Therefore we can define $\alpha=\alpha^{\prime} i d_{\mathcal{B}}$ to be an element of the wreath product $\hat{\mathbb{Z}}_{p}\left\langle\operatorname{Sym}(8)\right.$ and clearly $\alpha^{\prime} i d_{\mathcal{B}}$ is an element of $A$ with $\alpha^{\mathcal{B}}=\alpha^{\prime}$.

(b) Let us assume that $\Gamma_{0}$ is the empty graph.

Then Lemma 3.8(c) shows that every permutation in $\left\langle R_{1}, R_{2}\right\rangle^{(2)}$ lifts to an automorphism of $\Gamma$.

(c) Let us assume that $\Gamma_{0}$ is neither connected nor the empty graph.

Observation 4.2. If $R_{1} \neq R_{2}$, then $\langle\stackrel{\circ}{R}, \hat{R}\rangle \leq A$ contains $\beta_{1}, \beta_{2}, \beta_{3}$ such that

$$
\beta_{1}^{\mathcal{B}}=\left(B_{1} B_{2}\right)\left(B_{3} B_{4}\right), \beta_{2}^{\mathcal{B}}=\left(B_{1} B_{2}\right)\left(B_{5} B_{6}\right), \beta_{3}^{\mathcal{B}}=\left(B_{1} B_{2}\right)\left(B_{7} B_{8}\right) .
$$

Proof. Recall from Lemma 3.5 that $\langle\hat{R}, \stackrel{\circ}{R}\rangle$ is the same group whether $R$ is $Q$ or $\mathbb{Z}_{2}^{3}$. By Lemma 3.4 the elements $\beta_{1}, \beta_{2}, \beta_{3}$ can be generated as products of an element of $\stackrel{\circ}{R}$ and $\hat{R}$, as in the proof of Proposition 3.6, if $R=Q$.

Lemma 4.3. We claim that $B_{2 k-1}$ and $B_{2 k}$ are in the same connected component of $\Gamma_{0}$ for $k=1,2,3,4$.

Proof. Since $\Gamma_{0}$ is a Cayley graph and $R_{1}$ is transitive on the pairs of the form $\left(B_{2 k-1}, B_{2 k}\right)$ it is enough to prove that $B_{1}$ and $B_{2}$ are in the same connected component of $\Gamma_{0}$. If $B_{1} \nsim B_{2}$, then $B_{1}$ is adjacent to $B_{2}$ in $\Gamma_{0}$, so we can assume that $B_{1} \sim B_{2}$. Since $\Gamma_{0}$ is not the empty graph $B_{1}$ is adjacent to $B_{l}$ for some $l>2$, so $B_{1} \nsim B_{l}$. By Observation (4.2) there exists $\beta \in A$ such that $\beta\left(B_{1}\right)=B_{2}$ and $\beta\left(B_{l}\right)=B_{l}$. This shows that $B_{2} \nsim B_{l}$ and hence there is a path from $B_{1}$ to $B_{2}$ in $\Gamma_{0}$.

$\Gamma_{0}$ is not connected, so the order of the connected components of $\Gamma$ cannot be bigger than 4. Since $B_{1}$ and $B_{2}$ are in the same connected component of $\Gamma_{0}$ there exists a partition $H_{1} \cup H_{2}=\mathcal{B}$ such that $\left|H_{1}\right|=\left|H_{2}\right|=4, B_{1}, B_{2} \in H_{1}$ and no vertex in $H_{1}$ is adjacent to any vertex of $H_{2}$ in $\Gamma_{0}$.

Lemma 4.4. There exists $\alpha \in A$ such that $\alpha^{\mathcal{B}}=\alpha^{\prime}$.

Proof. Let us assume first that $H_{1}=\left\{B_{1}, B_{2}, B_{3}, B_{4}\right\}$. Then we define $\alpha_{1}$ to be equal to $\beta_{2}$ on $H_{1}$ and the identity on $H_{2}$, where $\beta_{2}$ is defined in Observation 4.2. Using Lemma 3.8(c) we get that $\alpha_{1}$ is in $\langle\stackrel{\circ}{R}, \hat{R}\rangle^{(2)}$.

If $H_{1}=\left\{B_{1}, B_{2}, B_{5}, B_{6}\right\}$ or $H_{1}=\left\{B_{1}, B_{2}, B_{7}, B_{8}\right\}$, then we define $\alpha_{2}$ by $\left.\alpha_{2}\right|_{H_{1}}=\beta_{1}$ and $\left.\alpha_{2}\right|_{H_{2}}=i d$, where $\beta_{1}$ is defined in Observation 4.2. Lemma 3.8(c) shows again that $\alpha_{2} \in A$.

It is easy to see that $\alpha_{1}^{\mathcal{B}}=\alpha_{2}^{\mathcal{B}}=\left(B_{1} B_{2}\right)$. Therefore $A$ contains an element $\alpha$ such that $R_{1}^{\alpha^{\mathcal{B}}}=R_{2}$.

We conclude that we can assume that $R_{1}=R_{2}$. 


\subsection{Step 3}

Let us now assume that $R_{1}=R_{2}$. We intend to find $\gamma \in A$ such that $\stackrel{\circ}{R}^{\gamma}=\hat{R}$.

Let $\hat{x}$ and $\stackrel{\circ}{x}$ denote the generators of $\hat{\mathbb{Z}}_{p}$ and $\stackrel{\circ}{\mathbb{Z}}_{p}$, respectively. We may assume that $\left.\hat{x}\right|_{B_{1}}=\left.\stackrel{\circ}{x}\right|_{B_{1}}$.

Lemma 4.5. There exists $\gamma \in A$ such that $\stackrel{\circ}{x}^{\gamma}=\hat{x}$.

Proof. Let us assume first that $\Gamma_{0}$ is connected. It is clear by Lemma 3.8 (b) that $\stackrel{\circ}{x}=\hat{x}$. So, we may take $\gamma=1$.

Let us assume that $\Gamma_{0}$ is not connected. In this case there are at least two connected components which we denote by $\mathscr{C}_{1}, \ldots, \mathscr{C}_{n}$. We may assume that $B_{1} \in \mathscr{C}_{1}$. The permutations $\hat{x}$ and $\stackrel{\circ}{x}$ are elements of the base group of $\hat{\mathbb{Z}}_{p}<\operatorname{Sym}(8)$ and hence they can be considered as functions on $\mathcal{B}$. We may assume that $\hat{x}(r, u)=(r, u+1)$ for every $(r, u) \in R \times \mathbb{Z}_{p}$. By Lemma 3.8 (b), the function $\stackrel{\circ}{x}$ is constant on each equivalence class.

For every $1 \leq m \leq n$ there exists $\stackrel{\circ}{r}_{m} \in \stackrel{\circ}{R}$ such that $\stackrel{\circ}{r}_{m}\left(\mathscr{C}_{1}\right)=\mathscr{C}_{m}$ and for every $\stackrel{\circ}{r}_{m} \in \stackrel{\circ}{R}$ there exists $\hat{r}_{m} \in \hat{R}$ such that $\stackrel{\stackrel{\mathcal{H}}{\mathcal{B}}}{m}^{\mathrm{B}} \hat{r}_{m}^{\mathcal{B}}$. Let $\gamma$ be defined as follows:

$$
\begin{aligned}
\gamma \mid \cup \mathscr{C}_{1} & =i d \\
\gamma \mid \cup \mathscr{C}_{m} & =\stackrel{\circ}{m}_{m} \hat{r}_{m}^{-1} \text { for } 2 \leq m \leq n .
\end{aligned}
$$

Let $(b, v) \in \stackrel{\circ}{r}_{m}\left(B_{e}\right)$ with $B_{e} \in \mathscr{C}_{1}$ and we denote $\stackrel{\circ}{r}_{m}^{-1}(b, v)$ by $(a, u)$. Since $\stackrel{\circ}{x}$ is constant on $\mathscr{C}_{m}$ we have $\stackrel{x}{x}^{s}(b, v)=\left(b, v+c_{m} s\right)$ for some $c_{m}$ which only depends on $\mathscr{C}_{m}$. Thus $\stackrel{\circ}{r}_{m}(a, u+s)=\left(b, v+c_{m} s\right)$ since $\stackrel{\circ}{x}$ and $\stackrel{\circ}{r}_{m}$ commute and $\left.\stackrel{\circ}{x}\right|_{B_{e}}=\left.\hat{x}\right|_{B_{e}}$. Therefore we have

$$
\gamma(b, w)=\stackrel{\circ}{r}_{m}(a, w)=\stackrel{\circ}{r}_{m}(a, u+(w-u))=\left(b, v+c_{m}(w-u)\right)
$$

for every $(b, w) \in \stackrel{\circ}{r}_{m}\left(B_{e}\right)$. It is easy to verify that $\gamma^{-1}(b, w)=\left(b, \frac{w-v+u c_{m}}{c_{m}}\right)$ for every $w \in \mathbb{Z}_{p}$ which gives

$\gamma^{-1} \stackrel{\circ}{x}(b, w)=\gamma^{-1} \stackrel{\circ}{x}\left(b, w c_{m}+v-u c_{m}\right)=\gamma^{-1}\left(b, w c_{m}+v-u c_{m}+c_{m}\right)=(b, w+1)$.

It follows that $\gamma^{-1} \stackrel{\circ}{x} \gamma=\hat{\gamma}$.

It remains to show that $\gamma \in A$. Let $y$ and $z$ be two elements of $R \times \mathbb{Z}_{p}$.

We denote by $B_{y}$ and $B_{z}$ the elements of $\mathcal{B}$ containing $y$ and $z$, respectively. If $B_{y}$ and $B_{z}$ are in the same connected component of $\Gamma_{0}$, then either $\gamma$ is defined on $B_{y}$ and $B_{z}$ by $\stackrel{\circ}{r}_{m} \hat{r}_{m}^{-1}$ which is the element of the group $\langle\stackrel{G}{G}, \hat{G}\rangle \leq A$ or $\gamma(y)=y$ and $\gamma(z)=z$.

If $B_{y}$ and $B_{z}$ are not in the same connected component, then $B_{y} \sim B_{z}$. The definition of $\gamma$ shows that $\gamma^{\mathcal{B}}=i d$. Using Lemma 3.8 (c) we get that $\left.\gamma\right|_{B_{y} \cup B_{z}}$ is an automorphism of the induced subgraph of $\Gamma$ on the set $B_{y} \cup B_{z}$, which proves that $\gamma \in A$, finishing the proof of Lemma 4.5.

Using Lemma 4.5 we may assume that $\stackrel{\circ}{x}=\hat{x}$. Since $\stackrel{\circ}{x}$ and $\stackrel{\circ}{r}$ commute we have $\stackrel{\circ}{R} \times \stackrel{\circ}{\mathbb{Z}}_{p} \leq \hat{\mathbb{Z}}_{p} 2 \operatorname{Sym}(8)$. Now we can apply Lemma 4.2 which gives $(\stackrel{\circ}{r})_{b}=i d$ for every $\stackrel{\circ}{r} \in \stackrel{\circ}{R}$. This proves that $\stackrel{\circ}{R}=\hat{R}$ since $R_{1}=R_{2}$. Therefore $\stackrel{\circ}{G}=\hat{G}$, finishing the proof of Proposition 4.1.

It is straightforward to check that the proof of Proposition 4.1 only uses the fact that $p>8$ in the first step of the argument. We can formulate this fact in Proposition 4.6. 
Proposition 4.6. Let $\Gamma$ be a Cayley graph of $G=Q \times \mathbb{Z}_{p}$ or $G=\mathbb{Z}_{2}^{3} \times \mathbb{Z}_{p}$, where $p$ is an odd prime and let $\stackrel{\circ}{G}=\stackrel{\circ}{Q} \times \stackrel{\mathbb{Z}}{p}_{p}$ or $\stackrel{\circ}{G}=\stackrel{\circ}{\mathbb{Z}}_{2}^{3} \times \stackrel{\circ}{\mathbb{Z}}_{p}$ be a regular subgroup of $A$ ut $(\Gamma)$ isomorphic to $G$. Let us assume that there exists $a\langle\hat{G}, \stackrel{\leftrightarrow}{G}\rangle$-invariant partition $\mathcal{B}=\left\{B_{1}, B_{2}, \ldots, B_{8}\right\}$ of $V(\Gamma)$, where $\left|B_{i}\right|=p$ for every $i=\{1, \ldots, 8\}$. In addition, we assume that $\stackrel{\mathbb{Z}}{p}_{p}$ is a subgroup of the base group of $\hat{\mathbb{Z}}_{p} 2 \operatorname{Sym}(\mathcal{B})$. Then there is an automorphism $\alpha$ of the graph $\Gamma$ such that $\hat{G}^{\alpha}=\stackrel{\circ}{G}$.

\section{Main result for $\boldsymbol{p}=5$ and 7}

In this section we will prove that $Q \times \mathbb{Z}_{5}, Q \times \mathbb{Z}_{7}, \mathbb{Z}_{2}^{3} \times \mathbb{Z}_{5}$ and $\mathbb{Z}_{2}^{3} \times \mathbb{Z}_{7}$ are CI-groups.

The whole section is based on the paper [5], so we will only modify the proof of Lemma 5.4 of [5].

Proposition 5.1. Every Cayley graph of $Q \times \mathbb{Z}_{5}, Q \times \mathbb{Z}_{7}, \mathbb{Z}_{2}^{3} \times \mathbb{Z}_{5}$ and $\mathbb{Z}_{2}^{3} \times \mathbb{Z}_{7}$ is a CI-graph.

We let $R$ denote either $Q$ or $\mathbb{Z}_{2}^{3}$, and let $p=5$ or 7 . Let $\Gamma$ be a Cayley graph of $R \times \mathbb{Z}_{p}$ and let $A=A u t(\Gamma)$. We denote by $P$ a Sylow $p$-subgroup of $A$. Let us assume that $A$ contains two copies of regular subgroups which we denote by $\hat{G}=\hat{R} \times \hat{\mathbb{Z}}_{p}$ and $\stackrel{\circ}{G}=\stackrel{\circ}{R} \times \stackrel{\circ}{\mathbb{Z}}_{p}$. We can assume that $\Gamma$ is neither the empty nor the complete graph and both $\hat{\mathbb{Z}}_{p}$ and $\stackrel{\circ}{\mathbb{Z}}_{p}$ are contained in $P$.

If the order of every orbit of $P$ on $V(\Gamma)$ is $p$, then it is clear from Proposition 4.6 that $\Gamma$ is a CI-graph. Therefore $P$ has an orbit $\Lambda \subset G$ such that $|\Lambda|=p^{2}$ since $p^{3}>|G|$. The remaining orbits of $P$ have order $p$ since $2 p^{2}>8 p$.

It was proved in [5] Lemma 5.4 that the action of $A$ on the vertices of the graph $\Gamma$ cannot be primitive so there is a nontrivial $A$-invariant partition $\mathcal{B}=\left\{B_{0}, B_{1}, \ldots, B_{t-1}\right\}$ of $V(\Gamma)=G$. The elements of the partition $\mathcal{B}$ have the same cardinality since the action of $A$ is transitive on $\mathcal{B}$ so $\left|B_{i}\right| \leq 4 p<p^{2}$ for every $i=0,1, \ldots, t-1$. The partition $\mathcal{B}$ is $P$-invariant so $P$ acts on $\mathcal{B}$. Since $P$ is a $p$-group, the order of every orbit of $P$ is a power of $p$.

Let $\mathscr{C}=\left\{C_{0}, C_{1}, \ldots, C_{s-1}\right\}$ be an orbit of $P$ on $\mathcal{B}$ such that $\Lambda \subseteq \cup_{i=0}^{s-1} C_{i}$. We may assume that $B_{i}=C_{i}$ for $i=0,1, \ldots, s-1$. It is clear that $s$ is a power of $p$. If $s \geq p^{2}$, then $\left|\cup_{i=0}^{s-1} C_{i}\right| \geq 2 p^{2}>8 p$ which is a contradiction. Since $\left|C_{0}\right|=\left|B_{0}\right|<p^{2}$, we cannot have $s=1$. It follows that $1<s<p^{2}$ which implies $s=p$.

For every $i<s$ and every $x \in P$ the following eqalities hold for some $j<s$

$$
x\left(B_{i} \cap \Lambda\right)=x\left(B_{i}\right) \cap x(\Lambda)=B_{j} \cap \Lambda .
$$

This implies that

$$
\left|B_{0} \cap \Lambda\right|=\left|B_{i} \cap \Lambda\right|
$$

for every $0 \leq i<s$. Therefore

$$
p^{2}=|\Lambda|=\left|\cup_{i=0}^{s-1}\left(B_{i} \cap \Lambda\right)\right|=s\left|B_{0} \cap \Lambda\right|=p\left|B_{0} \cap \Lambda\right| .
$$

This gives $\left|B_{0} \cap \Lambda\right|=p$ so $\left|B_{0}\right|$ can only be $p$ or 8 since $\left|B_{0}\right| t=8 p$ and both $\left|B_{0}\right|$ and $t \geq s$ are at least $p$.

If $\left|B_{0}\right|=p$, then $\Lambda$ is the union of $p$ elements of the $A$-invariant partition $\mathcal{B}$ and every orbit $\Lambda^{\prime}$ of $P$ is an element of the partition $\mathcal{B}$ if $\Lambda^{\prime} \neq \Lambda$. For every orbit $\Lambda^{\prime} \neq \Lambda$ of $P$ and 
for every $y \in \hat{\mathbb{Z}}_{p} \cup \stackrel{\mathbb{Z}}{p}_{p}$ we have $y\left(\Lambda^{\prime}\right)=\Lambda^{\prime}$. In particular, $y\left(B_{7}\right)=B_{7}$. By Proposition 4.6 we may assume that there exists an element $x^{\prime}$ in $\hat{\mathbb{Z}}_{p} \cup \stackrel{\mathbb{Z}}{p}_{p}$ such that $x^{\prime}\left(B_{0}\right) \neq B_{j}$ for some $j \neq 0,7$ and clearly $x^{\prime}\left(B_{7}\right)=B_{7}$. Since both $\hat{G}$ and $\dot{G}$ are regular there exists $a \in C_{A}\left(x^{\prime}\right)$ such that $a\left(B_{0}\right)=B_{7}$. Since $a$ and $x^{\prime}$ commute we have $a\left(B_{j}\right)=B_{7}$, which contradicts the fact that $a\left(B_{0}\right)=B_{7}$.

We must therefore have $\left|B_{0}\right|=8$. Let $\hat{x}$ and $\stackrel{\circ}{x}$ generate $\hat{\mathbb{Z}}_{p}$ and $\stackrel{\circ}{\mathbb{Z}}_{p}$, respectively. Since $\hat{G}$ and $\stackrel{\circ}{G}$ are regular we have that neither $\hat{x}^{\mathcal{B}}$ nor $\dot{x}^{\mathcal{B}}$ is the identity, so both $\hat{x}$ and $\stackrel{\circ}{x}$ are regular on $\mathcal{B}$. Since both $\hat{x}^{\mathcal{B}}$ and $\dot{x}^{\mathcal{B}}$ generate a transitive subgroup in $\operatorname{Sym}(\mathcal{B})$ of prime order $p>2$, and every for $r \in \hat{R} \cup \stackrel{\circ}{R}$ the permutation $r^{\mathcal{B}}$ commutes with one of these two elements, we have $r^{\mathcal{B}}=i d$. Since $\hat{x}$ and $\stackrel{x}{x}$ are in the same Sylow $p$-subgroup of $P$ we may assume that $\hat{x}\left(B_{i}\right)=\stackrel{\circ}{x}\left(B_{i}\right)=B_{i+1}$ for $i=0,1, \ldots, p-1$, where the indices are taken modulo $p$. By Proposition 4.6 we may also assume that $\hat{x} \neq \stackrel{\circ}{x}$.

For every $m$ there exists an $l$ such that the action of $\hat{x}^{l} \stackrel{\circ}{x}^{-l}$ is nontrivial on $B_{m}$ since $\hat{x} \neq \stackrel{\circ}{x}$. Therefore $\left.A_{B_{m}}\right|_{B_{m}}$ contains a regular subgroup and a cycle of length $p$ such that $p>\frac{\left|B_{0}\right|}{2}$. A theorem of Jordan on primitive permutation groups, which can also be found in [8, Theorem 13.1.], says that such a permutation group is 2-transitive and hence the induced subgraph of $\Gamma$ on $B_{m}$ is the complete or the empty graph for every $m$.

Lemma 5.2. $B_{m} \sim B_{n}$ for $0 \leq m<n \leq p-1$.

Proof. There exists a unique element $\hat{g} \in \hat{\mathbb{Z}}_{p} \leq P$ such that $\hat{g}\left(B_{m}\right)=B_{n}$. We also have a unique element $\stackrel{\circ}{g} \in \stackrel{\circ}{\mathbb{Z}}_{p} \leq P$ with $\hat{g}^{\mathcal{B}}=\stackrel{\circ}{g}^{\mathcal{B}}$. Since $\mathbb{Z}_{p}$ is a cyclic group of prime order and

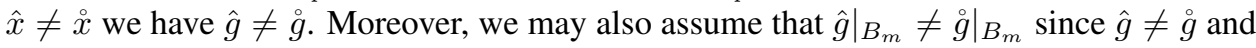
the induced subgraphs of $\Gamma$ on $B_{m+c} \cup B_{n+c}$ are all isomorphic, where both $m+c$ and $n+c$ are taken modulo $p$.

Clearly, $\tilde{g}=\stackrel{\circ}{g} \hat{g}^{-1}$ is a cycle of length $p$ on $B_{n}$. The vertices of $V(\Gamma) \backslash \Lambda$ are contained in $P$-orbits of order $p$ that contain the orbit of the vertex under $x$, so meet each $B_{i}$ in a single vertex, so $\tilde{g}$ fixes every vertex of the set $B_{m} \cup B_{n} \backslash \Lambda$ since $\tilde{g}^{\mathcal{B}}=i d$.

Let $u \in B_{m} \backslash \Lambda$. It is enough to show that if $u$ is adjacent to some $v \in B_{n}$, then $u$ is adjacent to every vertex of $B_{n}$. We will prove that $A$ is transitive on the following pairs: $\left\{(u, w) \mid w \in B_{n}\right\}$.

$A$ is transitive on $\left\{(u, w) \mid w \in B_{n} \cap \operatorname{supp}(\tilde{g})\right\}=\left\{(u, w) \mid w \in B_{n} \cap \Lambda\right\}$ since $\tilde{g}$ fixes $u$. Therefore we may assume that $v \in B_{n} \backslash \Lambda$ and we only have to find an element $a \in A$ such that $a(u)=u$ and $a(v) \in B_{n} \cap \Lambda$.

The restriction of $\tilde{g}$ to $B_{n}$ is a cycle of length $p$ so $\tilde{g}$ does not commute with $\left.\stackrel{r}{r}\right|_{B_{n}}$, where $\stackrel{\circ}{r}$ is an involution of $\stackrel{\circ}{R}$. Since $\stackrel{\circ}{r}$ and $\stackrel{\circ}{g}$ commute we have that there is a $u^{\prime} \in B_{m}$ such that $\stackrel{r}{r} \hat{g}\left(u^{\prime}\right) \neq \hat{g} \stackrel{\circ}{r}\left(u^{\prime}\right)$. Since the action of $\hat{R}$ is transitive on $B_{m}$ there exists $\hat{r} \in \hat{R}$ such that $\hat{r}(u)=u^{\prime}$. Then

$$
(\stackrel{\circ}{r} \hat{r}) \hat{g}(u)=\stackrel{\circ}{r} \hat{r} \hat{r}(u)=\stackrel{\circ}{r} \hat{g}\left(u^{\prime}\right) \neq \hat{g} \stackrel{\circ}{r}\left(u^{\prime}\right)=\hat{g}(\stackrel{\circ}{r} \hat{r})(u)
$$

so there exists $a^{\prime} \in A$ such that

$$
a^{\prime} \hat{g}(u) \neq \hat{g} a^{\prime}(u)
$$

Let us suppose that $v=\hat{g}(u)$. Notice that $\hat{g}(u)$ is in a $P$-orbit of order $p$, so $\hat{g}(u) \notin \Lambda$. Then the inequality (5.1) gives $a^{\prime}(v) \neq \hat{g} a^{\prime}(u)$. Since $\left.\hat{R}\right|_{B_{m}}$ is regular on $B_{m}$ there exists $\hat{s} \in \hat{R}$ such that $\hat{s}(u)=a^{\prime}(u)$ and since $\hat{s}$ and $\hat{g}$ commute we have $\hat{s}(v)=\hat{s} \hat{g}(u)=$ $\hat{g} \hat{s}(u)=\hat{g} a^{\prime}(u)$. Therefore $\hat{s}(v) \neq a^{\prime}(v)$ and hence $\hat{s}^{-1} a^{\prime}$ fixes $u$ and $\hat{s}^{-1} a^{\prime}(v) \neq v$ so we may assume that $v \neq \hat{g}(u)$. 
If $p=7$, then $v \in B_{n} \cap \Lambda$.

Let us assume that $p=5$. We claim that there exists $\hat{t} \in \hat{R}$ such that $\hat{t}(u) \in B_{m} \backslash \Lambda=$ $B_{m} \backslash \operatorname{supp}(\tilde{g})$ while $\hat{t}(v) \in B_{n} \cap \Lambda=B_{n} \cap \operatorname{supp}(\tilde{g})$. It is clear that $\hat{g}\left(B_{m} \cap \operatorname{supp}(\tilde{g})\right)=$ $B_{n} \cap \operatorname{supp}(\tilde{g})$ and $\hat{g}$ commutes with each element of $\hat{R}$. Therefore it is enough to show that if $u, v \in B_{m} \backslash \operatorname{supp}(\tilde{g})$ with $u \neq v$, then there exists $\hat{t} \in \hat{R}$ such that $\hat{t}(u) \in B_{m} \backslash \operatorname{supp}(\tilde{g})$ and $\hat{t}(v) \in B_{m} \cap \operatorname{supp}(\tilde{g})$. This can easily be seen from the fact that $\operatorname{gcd}(|\hat{R}|, 5)=1$.

The permutations $\hat{t}^{-1} \tilde{g}^{l} \hat{t}$ fix the vertex $u$ for every $0 \leq l \leq 4$ and $\hat{t}^{-1} \tilde{g}^{l_{1}} \hat{t}(v) \neq$ $\hat{t}^{-1} \tilde{g}^{l_{2}} \hat{t}(v)$ if $l_{1} \not \equiv l_{2}(\bmod p)$. At least one of the the four elements $\hat{t}^{-1} \tilde{g} \hat{t}, \hat{t}^{-1} \tilde{g}^{2} \hat{t}, \hat{t}^{-1} \tilde{g}^{3} \hat{t}$, $\hat{t}^{-1} \tilde{g}^{4} \hat{t}$ of $A$ fixes $u$ and maps $v$ to an element of $B_{n} \cap \operatorname{supp}(\tilde{g})=B_{n} \cap \Lambda$ since $\left|B_{n}\right|$ $\operatorname{supp}(\tilde{g}) \mid=3$, finishing the proof of the fact that $B_{m} \sim B_{n}$ for $0 \leq m \neq n \leq 7$.

Every permutation of $V(\Gamma)$ which fixes $B_{m}$ setwise for every $m$ is an automorphism of $\Gamma$ so there is an $a \in A$ such that $\dot{x}^{a}=\hat{x}$. Applying Proposition 4.6 we get that there exists $\alpha \in A$ such that $\left(\hat{R} \times \hat{\mathbb{Z}}_{p}\right)^{\alpha}=\stackrel{\circ}{R} \times \stackrel{\circ}{\mathbb{Z}}_{p}$, finishing the proof of Proposition 5.1.

\section{References}

[1] L. Babai, Isomorphism problem for a class of point-symmetric structures, Acta Math. Acad. Sci. Hungar. 29 (1977), 329-336.

[2] E. Dobson, The isomorphism problem for Cayley ternary relational structures for some abelian groups of order 8p, Dicrete Math. 310 (2010), 2895-2909.

[3] E. Dobson, P. Spiga, CI-groups with respect to ternary relational structures: new examples, Ars Math. Contemp. 6 (2012), 351-364.

[4] C. H. Li, On isomorphisms of finite Cayley graphs- a survey, Discrete Mathematics 256 (2002), 301-334.

[5] C. H. Li, Z. P. Lu, P. P. Pálfy, Further restrictions on the structure of finite CI-groups, J. Algebr. Comb. (2007), 161-181.

[6] P. Spiga, On the Cayley isomorphism problem for a digraph with 24 vertices, Ars. Math. Contemp. 1 (2008), 38-43.

[7] G. Royle, Constructive enumeration of graphs, Thesis submitted to The University of Western Australia, 1987.

[8] H. Wielandt, Finite permutation groups, Academic Press, London-New York, 1964. 